(C) 2016 IEEE. Personal use of this material is permitted. Permission from IEEE must be obtained for all other uses, in any current or future media, including reprinting/republishing this material for advertising or promotional purposes, creating new collective works, for resale or redistribution to servers or lists, or reuse of any copyrighted component of this work in other works. 


\title{
Wavelet PCA for Automatic Identification of Walking with and without an Exoskeleton on a Treadmill using Pressure and Accelerometer sensors
}

\author{
Ganesh R. Naik, Senior Member, IEEE, Gita Pendharkar, and Hung T. Nguyen, Senior Member, IEEE
}

\begin{abstract}
Nowadays portable devices with more number of sensors are used for gait assessment and monitoring for elderly and disabled. However, the problem with using multiple sensors is that if they are placed on the same platform or base, there could be cross talk between them, which could change the signal amplitude or add noise to the signal. Hence, this study uses wavelet PCA as a signal processing technique to separate the original sensor signal from the signal obtained from the sensors through the integrated unit to compare the two types of walking (with and without an exoskeleton). This comparison using wavelet PCA will enable the researchers to obtain accurate sensor data and compare and analyze the data in order to further improve the design of compact portable devices used to monitor and assess the gait in stroke or paralyzed subjects. The advantage of designing such systems is that they can also be used to assess and monitor the gait of the stroke subjects at home, which will save them time and efforts to visit the laboratory or clinic.
\end{abstract}

\section{INTRODUCTION}

Robotic exoskeleton has been gaining popularity and has helped many paralyzed subjects or stroke subjects to walk normally again through various training methods. Research carried out using robotic exoskeleton has also proved that regaining mobility through proper training can be achieved over a period of time. One of such robotic exoskeleton (ALEX), developed at the University of Delaware has been useful in training stroke subjects and after few weeks of training, the stroke subjects seem to start walking normally $[1,2]$. Although such robotic exoskeleton have the advantage of reducing the physical burden on clinical staff, however, while training the stroke subjects, they have to be physically attended by experts in order to use the robotic exoskeleton. The robotic exoskeleton could be bulky and needs to be attached to the waist or lower trunk in the correct manner so as not to cause discomfort to the subject. While such training is essential, once the subjects has regained a close to normal gait, they need continuous gait monitoring and assessment carried out regularly in order to make sure that they retain a normal heel-toe walking pattern.

To carry out gait assessment and monitoring in the laboratories, for such subjects, it becomes very difficult as they have to visit the clinic regularly in order to be assessed. Hence monitoring and assessing their gait at home or

Ganesh R. Naik, and Hung Nguyen are with Centre for Health Technologies, Faculty of Engineering and Information Technology (FEIT), University of Technology Sydney (UTS), NSW 2007, Australia. (e-mail: ganesh.naik@uts.edu.au and hung.nguyen@uts.edu.au).

Gita Pendharkar is with Electrical and Computer Systems Engineering Monash University, Melbourne, Australia. (e-mail: gita@monash.edu.au). remotely would be useful for the stroke subjects. Currently there is lot of research carried out in designing compact portable devices using accelerometers, gyroscopes or pressure sensors to assess and monitor their gait remotely. Literature review reveals that there is a lot of research carried out on gait analysis using wearable sensor system and identifying the various gait phases[2-5]. Inertial measurement unit (IMUs) are becoming more popular and commonly used for gait analysis in a number of applications [6, 7]. Some systems using multiple sensors such as a combination of IMUs and pressure sensors are also useful as they provide lot of information on different gait parameters being sensed. However, the problem with using multiple sensors in one system is that if they are placed on the same platform or base, there could be cross-talk between them which could change the signal amplitude or add noise to the signal.

In order to preserve the clinically valid information the signals must be well maintained during denoising process. A multivariate technique such as principal component analysis (PCA) is extensively used for such tasks. PCA has been applied for several engineering applications [7, 8]. For biomedical signals, a robust extension of classical PCA by utilising wavelets also known as multiscale PCA (MSPCA) is suggested [9-11]. This process has been successfully used for data reduction, classification and feature extraction $[8,12]$.

Firstly, the purpose of this research study was to look at the differences in the gait signals obtained using a multi sensor system (attached to the shoe) designed with IMU's of 6 degree of freedom (which integrates 3 axis accelerometers and 3 axis gyroscopes) and additionally four pressure sensors on the sole, when walking normally on a treadmill and walking with the robotic exoskeleton attached to the lower body as described in [1].

Secondly, this study uses wavelet PCA (MSPCA) as a signal processing technique to compare the two types of walking (with and without an exoskeleton) in order to separate the original sensor signal from the signal obtained from the sensors through the integrated unit. This comparison will enable the researchers to further improve the design of compact portable devices used to monitor and assess the gait in stroke or paralyzed subjects by implementing the correct gait features through software used to process the pressure and accelerometer data. The advantage of designing such systems is that they can also be used to assess and monitor the gait of the stroke subjects at home, which will save them time and efforts to visit the laboratory or clinic. 


\section{THEORY}

The Wavelet PCA or MSPCA combines the ability of PCA to decorrelate the variables by extracting a linear relationship, with that of wavelet analysis to extract deterministic features. MSPCA implements PCA to wavelet

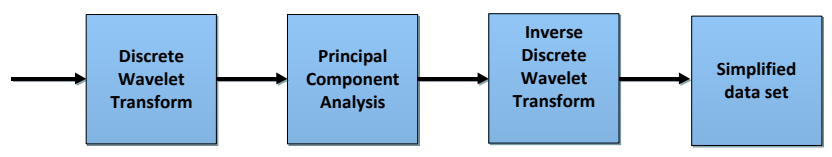

Figure 1. The overall schematic of Wavelet PCA

coefficients at each scale to filter the unwanted components $[13,14]$.

Wavelet PCA technique uses two major steps; the first one is wavelet decomposition of the individual sensors on a selected family of wavelets into approximations and details coefficients at different scales. In the second step, every sensor data from each scale are gathered as separate matrices and then, PCA is performed on the matrices of details of different levels and interesting simplified signals are reconstructed by retaining the numbers of principal components (PCs). Wavelet PCA is a powerful tool for monitoring autocorrelated measurements, and the method not only selects and monitors the significant signal information but also conforms to the nature of the signal [12-14]. The overall schematic of Wavelet PCA is shown in Figure 1.

\section{Methodology}

\section{A. Subjects}

The University Human Research Ethics Committee approved the experimental protocol for this study. A pilot study was carried out on healthy subjects. Data from four pressure sensors and three axis accelerometers was collected while walking normally on a treadmill with and without the exoskeleton attached. The error such as drift in accelerometers was minimized to zero. The data were collected using the robotic exoskeleton developed at the Department of Mechanical Engineering at the University of Delaware [1]. The subjects who volunteered to participate for the study were healthy and had no neurological, physical or vestibular disorders, which would affect their gait. The data were collected for five minutes of walking with and without the exoskeleton from five different subjects.

\section{B. Study design}

Pressure and accelerometer data were collected using a compact system made of IMU of six degrees of freedom (3 axis accelerometer, 3 axis gyroscopes) and four separate pressure sensors (placed on the sole). This compact system was interfaced to an Arduino Fio microcontroller as shown in Figure 2. The microcontroller was wirelessly interfaced to the receiver on a laptop which had Arduino Duo to carry out the basic sensor signal processing and stored the required information on the laptop for further signal processing. Safety standards were maintained while using the compact system on body.

\section{Experimental Setup and Data analysis}

The compact system was attached carefully on top of the shoe and tightened with the laces when the subject walked on the treadmill as shown in the Figure 3. There were two sets of experiments carried out using this compact system. For the first set of experiments, the subject walked on the treadmill for 5 minutes. The walking speed was chosen to be comfortable walking speed of $2.4 \mathrm{~m} / \mathrm{hr}$. For the second set of experiments, along with the compact system, the subject was attached to the robotic exoskeleton as shown in Figure 4 and walked at a speed of $2.4 \mathrm{~m} / \mathrm{hr}$. Before beginning each walking test, the subject was asked to stand still for one minute and the sensors were reset using the software.

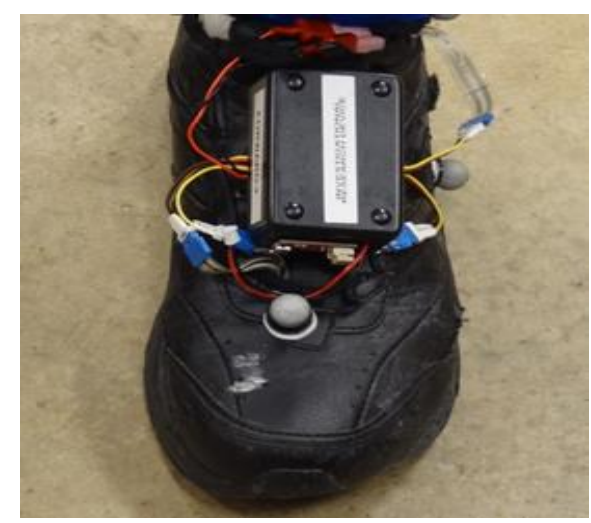

Figure 2. Compact System using Sensors

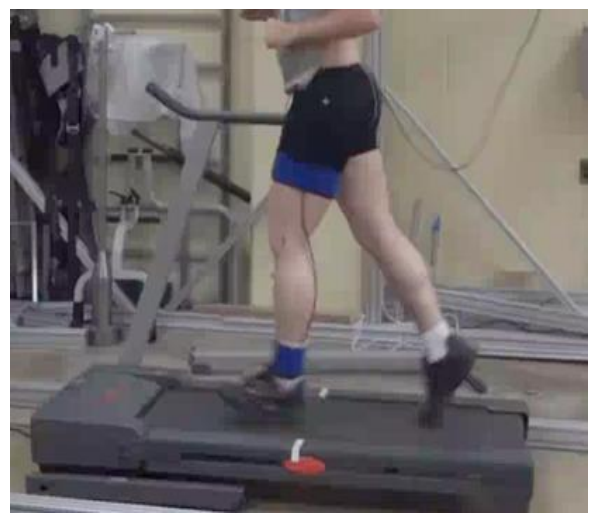

Figure 3. Walking on the treadmill with the compact sytem

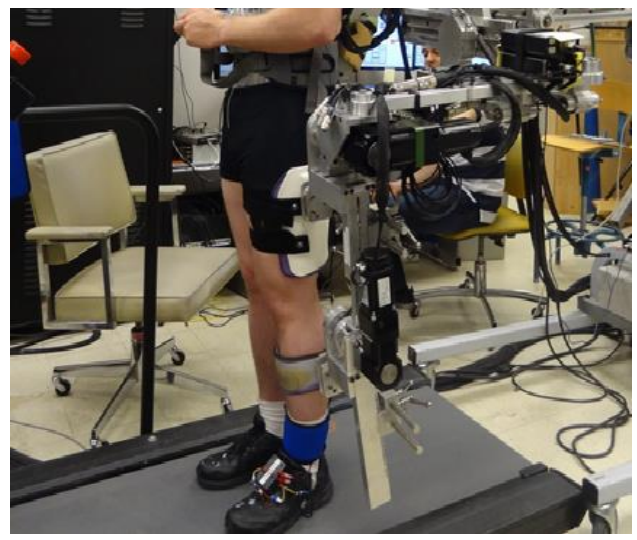

Figure 4. Walking with compact system and exoskeleton on treadmill 

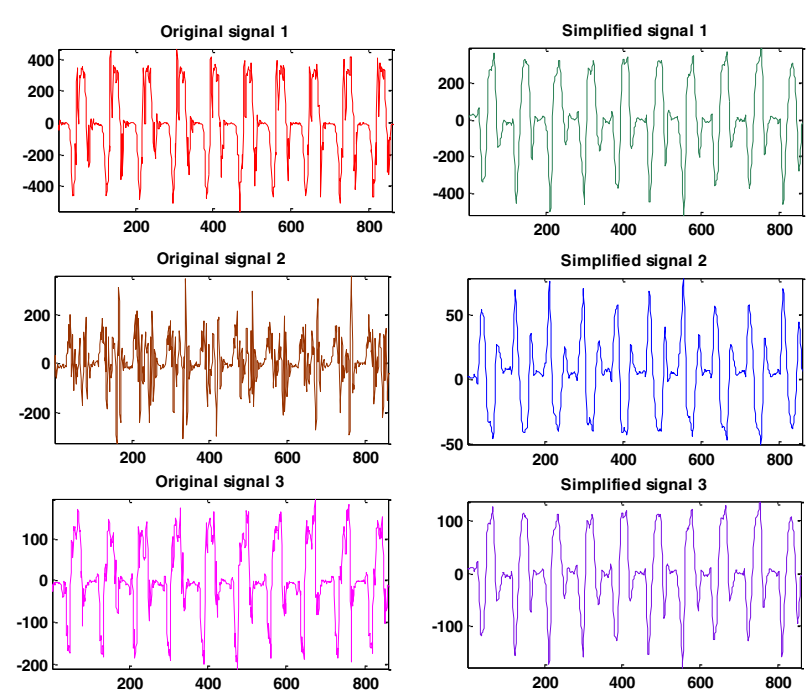

Figure 5. Accelormeter signals when walking normally with compact system.
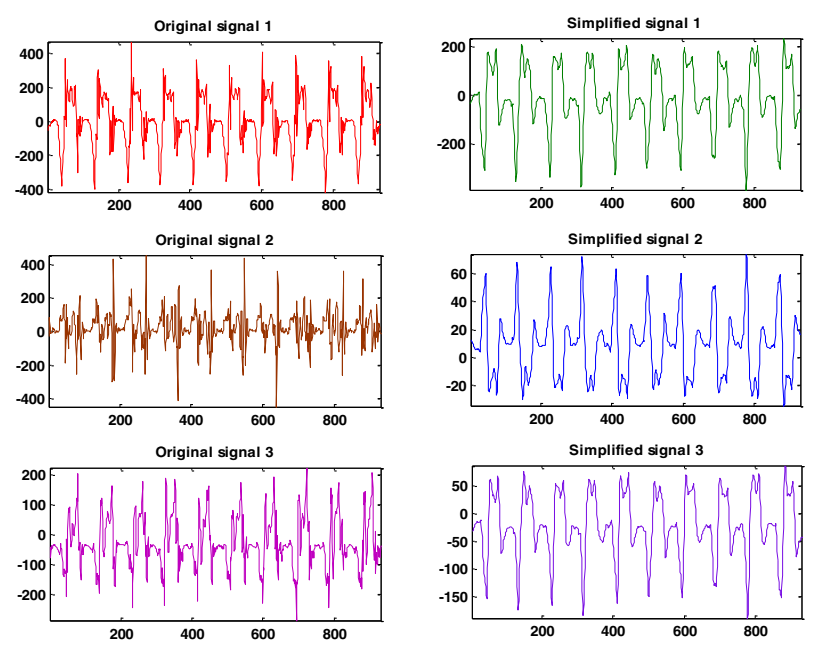

Figure 6. Accelrometer signals when walking with Exoskeleton and compact system.

The three accelerometer signals (x-mediolateral acceleration, y-horizontal or anterior-posterior acceleration, z-vertical or downwards acceleration) and four pressure signals (heel, toe, Lball, Mball) data were analyzed using custom Matlab software (Math Works, version 7.1). The vertical, and anterior-posterior, acceleration data were sampled at $40 \mathrm{~Hz}$ and then low pass filtered using 6th order Butterworth filter at $10 \mathrm{~Hz}$ cut-off frequency using a digital filter in Matlab.

In order to perform Wavelet PCA analysis, initially, appropriate wavelet parameters were set. Daubechies' leastasymmetric wavelet with 4 vanishing moments, "sym 4 " was used for this study [15]. In order to retain number of PCs Kaiser's rule was applied, which helps in retaining the number of PCs associated with eigenvalues exceeding the mean of all eigenvalues. The MSPCA algorithm with Kaiser' rules also reduce the noise and retain the PCs reflecting the quality of column reconstructions given by the relative mean square errors which are close to $100 \%[8]$.

\section{RESULTS AND DISCUSSIONS}

The original signals in Figure 5 and Figure 6 shows the mediolateral (original signal 1), vertical (original signal 2) and horizontal (original signal 3) acceleration signals when plotted w.r.t. time in Matlab. The simplified signals are the corresponding signals using MSPCA analysis. Figure 5 shows accelerometer signals when walking normally without the exoskeleton and Figure 6 shows the accelerometer signals (original and simplified using MSPCA) when walking with the exoskeleton.
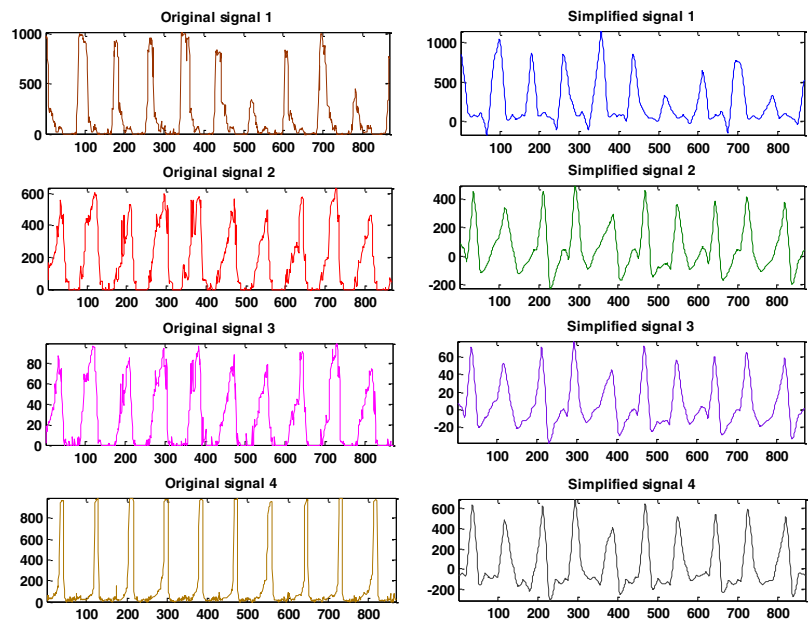

Figure 7. Force signals when walking normally with the compact system on treadmill.
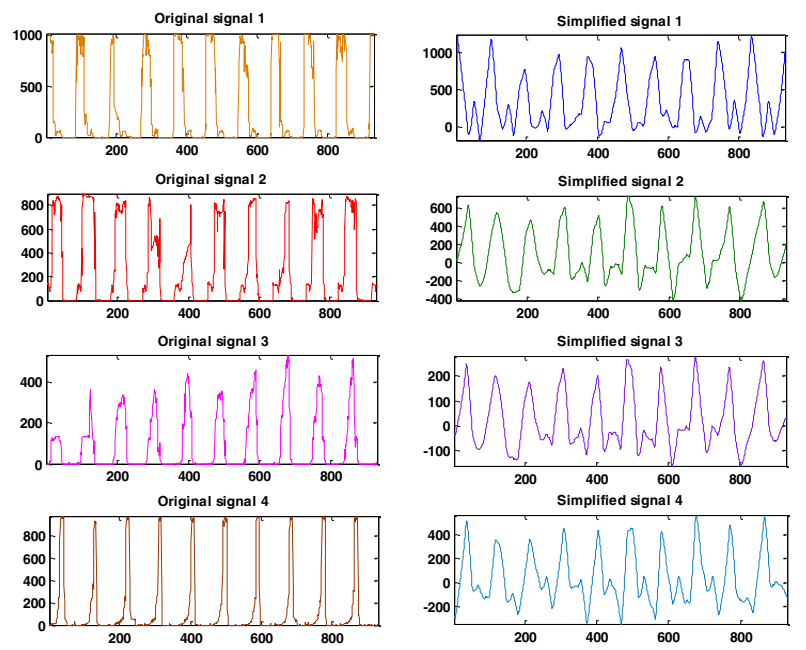

Figure 8. Force signals when walking with the Exoskelton and the compact system on treadmill.

Figures 7 and Figure 8 shows the force signals (in in $g-$ acceleration due to gravity) obtained from the pressure sensors plotted w.r.t. the sample numbers when walking with and without the exoskeleton. Signal 1 corresponds to heel pressure, signal 2 corresponds to toe pressure, signal 3 corresponds to lateral ball and signal 4 corresponds to medial ball pressure. Figure 7 shows pressure signals when walking normally without the exoskeleton and Figure 8 shows the pressure signals (original and simplified using MSPCA) when walking with the exoskeleton. 
From the results based on comparing Figures 5 and Figure 6, it can be observed that there are no significant differences in the accelerometer signals (original signal 1) when walking with and without the exoskeleton in the original signal 1 and simplified signal 2 (using MSPCA). However, when comparing the simplified signal 2 (vertical acceleration) in Figure 5 w.r.t the simplified signal 2 in Figure 6 , the mid stance phase seems to be slightly different as mentioned in $[2,16]$.

From the results based on Figures 7 and Figure 8, it can be observed that the peak value of the force exerted by the heel sensors (original signal 1) is approximately the same when walking with and without the exoskeleton. Although the heel pressure observed in simplified signal 2 (using MSPCA) in both the walking patterns shows few differences from the original signal 1, the peak amplitude of the force in both is the same. However, in case of the toe sensors (original signal 2), the peak value of the force exerted by toes is observed to be higher when walking with the exoskeleton than without the exoskeleton. This is also confirmed by observing the simplified signal 1 in Figure 7 and comparing it with simplified signal 1 from Figure 8, which is derived using MSPCA analysis. As all the four pressure sensors were placed on a single sole, there was a cross talk which was observed when we compare the force sensors in the original signal $1 \& 2$ with the simplified signals $1 \& 2$ in Figure 7 and Figure 8. Using MSPCA analysis on the original signal reduced this cross talk.

Derived from the normal walking acceleration signals from [8] we have the following equations during mid stance:

\section{$a_{v} \approx g$ where $a_{y}$ is the acceleration in vertical direction (original signal 2)}

$a_{h} \approx 0$ where $a_{h}$ is the acceleration in horizontal direction (original signal 3) \& $g=$ acceleration due to gravity

The vertical acceleration during the mid-stance in a gait cycle while walking without the exoskeleton corresponds to $g$ which is observed in the simplified signal 2 in Figure 5. But the simplified signal 2, in Figure 6, exhibits a more plantar flexed foot and higher peak values when walking with the exoskeleton attached which could be due to the loading effect caused due to the exoskeleton as mentioned in [17].

\section{CONCLUSION}

This study compared the two types of walking (with and without an exoskeleton) in order to separate the original sensor signal from the signal obtained from the sensors through the integrated unit. Wavelet PCA (MSPCA) was used as a signal processing tool for this task. It can also be concluded that the heel exerts the highest force when walking (with and without the exoskeleton) as compared to the toes, lateral and medial ball and this is because of the higher impact caused when the foot lands on the ground and has the heel contact. The peak value of the force exerted on toes was higher when walking with the exoskeleton (simplified signal 2, Figure 7) as compared to the force exerted on toes when walking without exoskeleton can be explained due to the loading effect caused due to the exoskeleton [17].

\section{ACKNOWLEDGMENT}

The authors would like to sincerely thank Prof. Sunil Agarwal, Damiano Zanotto \& the other staff members from the Mechanical Engineering Department at the University of Delaware for their support during the study.

\section{REFERENCES}

[1] S. K. Banala, S. H. Kim, S. K. Agrawal, and J. P. Scholz, "Robot assisted gait training with active leg exoskeleton (ALEX)," Neural Systems and Rehabilitation Engineering, IEEE Transactions on, vol. 17, pp. 2-8, 2009.

[2] S. H. Kim, S. K. Banala, E. A. Brackbill, S. K. Agrawal, V. Krishnamoorthy, and J. P. Scholz, "Robot-assisted modifications of gait in healthy individuals," Experimental brain research, vol. 202, pp. 809-824, 2010.

[3] R. Moe-Nilssen, "A new method for evaluating motor control in gait under real-life environmental conditions. Part 2: Gait analysis," Clinical Biomechanics, vol. 13, pp. 328-335, 1998.

[4] A. J. Padgaonkar, K. Krieger, and A. King, "Measurement of angular acceleration of a rigid body using linear accelerometers," Journal of Applied Mechanics, vol. 42, pp. 552-556, 1975.

[5] G. Pendharkar, S. Marin, R. Mayagoitia, and P. Percival, "Triaxial acclerometer pilot study designed to study the gait of vestibular patients," Australasian Physical \& Engineering Sciences in Medicine, vol. 30, p. 414, 2007.

[6] A. T. M. Willemsen, J. Van Alste, and H. Boom, "Real-time gait assessment utilizing a new way of accelerometry," Journal of Biomechanics, vol. 23, pp. 859-863, 1990.

[7] G. Pendharkar, G. R. Naik, A. Acharyya, and H. T. Nguyen, "Multiscale PCA to distinguish regular and irregular surfaces using tri axial head and trunk acceleration signals," in Engineering in Medicine and Biology Society (EMBC), 2015 37th Annual International Conference of the IEEE, 2015, pp. 4122-4125.

[8] J. Kevric and A. Subasi, "The Effect of Multiscale PCA De-noising in Epileptic Seizure Detection," Journal of medical systems, vol. 38, pp. 1-13, 2014.

[9] L. Sharma, S. Dandapat, and A. Mahanta, "Multichannel ECG data compression based on multiscale principal component analysis," Information Technology in Biomedicine, IEEE Transactions on, vol. 16, pp. 730-736, 2012.

[10] S. Xie, F. Jin, S. Krishnan, and F. Sattar, "Signal feature extraction by multi-scale PCA and its application to respiratory sound classification," Medical \& biological engineering \& computing, vol. 50, pp. 759-768, 2012.

[11] D. Widjaja, E. Vlemincx, and S. Van Huffel, "Multiscale principal component analysis to separate respiratory influences from the tachogram: Application to stress monitoring," in Computing in Cardiology (CinC), 2012, 2012, pp. 277-280.

[12] E. Gokgoz and A. Subasi, "Effect of multiscale PCA de-noising on EMG signal classification for diagnosis of neuromuscular disorders," Journal of medical systems, vol. 38, pp. 1-10, 2014.

[13] B. R. Bakshi, "Multiscale PCA with application to multivariate statistical process monitoring," AIChE journal, vol. 44, pp. 1596$1610,1998$.

[14] A. Akinduko and A. N. Gorban, "Multiscale principal component analysis," in Journal of Physics: Conference Series, 2014, p. 012081.

[15] S. Mohr, L. E. Ratcliff, P. Boulanger, L. Genovese, D. Caliste, T. Deutsch, and S. Goedecker, "Daubechies wavelets for linear scaling density functional theory," The Journal of chemical physics, vol. 140, p. 204110, 2014.

[16] G. Pendharkar, D. Morgan, and P. Percival, "Processing accelerometry data to detect stance phase in gait and differentiate toe-walking steps from normal steps in idiopathic toe-walking children," Intelligent sensors sensor network and information processing, 2005.

[17] A. Danielsson and K. S. Sunnerhagen, "Energy expenditure in stroke subjects walking with a carbon composite ankle foot orthosis," Journal of rehabilitation medicine, vol. 36, pp. 165-168, 2004. 